\title{
Automated Computation of One-loop Scattering Amplitudes
}

\section{Giovanni Ossola*}

New York City College of Technology (CUNY)

E-mail: gossola@citytech.cuny.edu

The problem of an efficient and automated computation of scattering amplitudes at the one-loop level for processes with more than 4 particles is crucial for the analysis of the LHC data. In this presentation I will review the main features of a powerful algorithm for the reduction of one-loop amplitudes that operates at the integrand level. The method, also known as OPP reduction, is an important building block towards a fully automated implementation of this type of calculations. I will describe some of the features of the existing numerical codes available for the reduction and discuss the ongoing efforts to target important issues such as stability, versatility and efficiency of the method.

13th International Workshop on Advanced Computing and Analysis Techniques in Physics Research February 22-27, 2010

Jaipur, India

* Speaker. 


\section{Motivation \& Introduction}

In order to properly understand the new data that will soon be collected by the experimental collaborations at the LHC, theory predictions are not reliable without accounting for higher orders. The discovery of new physics will only be possible if we have full control over the Standard Model backgrounds. Motivated by these challenging requirements, we observed great progress in our understanding of one-loop multi-leg scattering amplitudes [1].

In the last year NLO QCD corrections were calculated for several $2 \rightarrow 4$ processes of great importance for the physics of the LHC, such as $p p \rightarrow t \bar{t} b \bar{b}$ [2-4], $p p \rightarrow t \bar{t} j j$ [5], $W+3$ jet production [6-9], $Z+3$ jet production [10] as well as multi-gluon production [11-13].

The computation of one-loop cross sections is composed of two parts: the evaluation of virtual corrections, namely contributions obtained adding a virtual particle to the tree-order diagrams, and contributions from real emission of one additional particle in the initial and final states, required by the presence of soft and collinear singularities. There are several tools available to deal with real emission [14]: algorithms such as dipole subtraction [15] or the FKS residue subtraction [16] provided the background to develop powerful numerical tools [17-19] for NLO calculations.

In this presentation I will focus on algorithms and computational tools available to automatize the calculation of the virtual corrections. In particular, I will briefly discuss the numerical implementation of the OPP reduction technique. Different approaches to one-loop calculations and algebraic techniques were discussed in other presentations at this workshop [20-22]. A general overview on the importance of NLO computations for phenomenological studies and recent progress in their automated computation was presented by D. Maitre in a plenary talk [23].

\section{Calculation of NLO virtual corrections}

It is well known that the computation of the virtual one-loop part can be performed by decomposing the integral in terms of know scalar or tensorial master integrals, that are analytically known and can be computed by means of existing libraries, such as QCDloop [24,25] or OneLOop [26]. One can achieve this task following a fully analytic reduction process. The traditional PassarinoVeltman reduction [27,28], that proved its effectiveness for processes with a small number of particles, was limited for multi-leg applications by its algebraic complexity and the appearance of spurious singularities. Nevertheless, improved tensorial reduction methods led to the development of tools [29-31] that are able to deal efficiently and precisely with processes of high complexity such as $p p \rightarrow t \bar{t} b \bar{b}$ [3], or $q q \rightarrow b \bar{b} b \bar{b}$ [32], and tri-boson production [33,34].

In the last couple of years, inspired by unitarity arguments [35,36], alternative approaches have emerged whose aim is the direct determination of the coefficients of the scalar/tensorial one-loop master integrals. A new powerful framework for one-loop calculation was developed by merging the idea of employing the kinematic cuts of the scattering amplitudes with a careful analysis of the structure of the their integrand [37] contained in the so-called OPP method [38,39].

These new reduction techniques have been already implemented in various codes, such as CutTools [40], that is publicly available, BlackHat [41], and Rocket [42].

Respect to the improved tensorial reduction methods, the unitarity-based approaches require the calculation of a separate set of polynomial terms, known as rational terms, that arise naturally 
in an algebraic decomposition. The great advantage of these new techniques is their generality, since in principle they are applicable to all processes, regardless of their complexity. Also, as I will describe in the following sections, they are well suited for full automation. Numerical efficiency and the control over numerical instabilities, that are some of the strong features of purely algebraic techniques, will be important benchmarks to assess the level of maturity of the new methods, in particular if we want to apply them to processes with six and more particles.

\section{The OPP algorithm}

The OPP method, presented in Ref. [38, 39], employs a universal four-dimensional decomposition for the numerator of the integrand for any one-loop scattering amplitudes. This general structure can be written as:

$$
\begin{aligned}
N(q) & =\sum_{i_{0}<i_{1}<i_{2}<i_{3}}^{m-1}\left[d\left(i_{0} i_{1} i_{2} i_{3}\right)+\tilde{d}\left(q ; i_{0} i_{1} i_{2} i_{3}\right)\right] \prod_{i \neq i_{0}, i_{1}, i_{2}, i_{3}}^{m-1} D_{i}+\sum_{i_{0}<i_{1}<i_{2}}^{m-1}\left[c\left(i_{0} i_{1} i_{2}\right)+\tilde{c}\left(q ; i_{0} i_{1} i_{2}\right)\right] \prod_{i \neq i_{0}, i_{1}, i_{2}}^{m-1} D_{i} \\
& +\sum_{i_{0}<i_{1}}^{m-1}\left[b\left(i_{0} i_{1}\right)+\tilde{b}\left(q ; i_{0} i_{1}\right)\right] \prod_{i \neq i_{0}, i_{1}}^{m-1} D_{i}+\sum_{i_{0}}^{m-1}\left[a\left(i_{0}\right)+\tilde{a}\left(q ; i_{0}\right)\right] \prod_{i \neq i_{0}}^{m-1} D_{i}
\end{aligned}
$$

The quantities $d\left(i_{0} i_{1} i_{2} i_{3}\right)$ are the coefficients of 4-point scalar functions with denominators $D_{i}$ labeled by $i_{0}, i_{1}, i_{2}$, and $i_{3}$. In the same way, we call $c\left(i_{0} i_{1} i_{2}\right), b\left(i_{0} i_{1}\right), a\left(i_{0}\right)$ the coefficients of all possible 3-point, 2-point and 1-point scalar functions, respectively. The quantities $\tilde{d}, \tilde{c}, \tilde{b}, \tilde{a}$ are what we call "spurious" terms, i.e. terms that will vanish upon integration. These terms still depend on the integration momentum $q$ and their functional form is process-independent. Using Eq. (3.1), the task of computing the one-loop amplitude is reduced to the algebraic problem of extracting all the coefficients by evaluating the function $N(q)$ a sufficient number of times at different values of $q$. This is achieved very efficiently if we employ values of $q$ such that a subset of denominators $D_{i}$ vanish. More details about the reduction can be found in previous presentations [43].

To summarize, the strength of the method lies in the fact that the only information required in order to extract the coefficient of the master integrals is the knowledge of the numerical value of the numerator function for a finite set of values of the integration momentum, that correspond to complex poles of the denominators.

The OPP reduction algorithm has been implemented in the code CutTools [40], that is publicly available. In this approach, there are two sources of rational terms [44]: a first set $R_{1}$ appears from the mismatch in the dimensionality between reconstructed numerators and the $\mathrm{d}$ dimensionality of the master integrals. This part can be recovered automatically by evaluating the amplitudes for a shifted value of the mass. A second set $R_{2}$ comes from the d-dimensionality of the numerator function: this part is recovered by means of ad hoc treelevel-like Feynman rules, that are provided in Refs. [44-46], for QED, QCD and the full Standard Model respectively.

The OPP method does not provide any specific recipe for the generation of the numerator function. Some of the calculations based on CutTools that appeared in the literature $[47,48]$ employ traditional Feynman diagrams. More recent and challenging calculations [4,5], evaluate the numerator numerically by means of HELAC-1LOOP [49].

A very interesting extension of the OPP method has been presented by Ellis, Giele, Kunszt and Melnikov [50]. In their approach, the reconstruction of the numerator is performed directly in 
d-dimensions. This framework is usually known as generalized d-dimensional unitarity [51, 52]. Further technical improvements in this direction, together with physical results for the process $p p \rightarrow t \bar{t} j$, have been recently presented by Melnikov and Schulze [53].

A new tool for the numerical computation of one-loop amplitudes has been released very recently [54]. It relies on the extension of the OPP-polynomial structures to include an explicit dependence on the extra-dimensional parameter and makes use of a polynomial interpolation based on the techniques described in [55].

\section{Generation of Amplitudes and Numerical Tests}

In this Section we will focus on the generation of unintegrated amplitudes (or numerator functions). Methods based on generalized unitarity provide to the reduction algorithm tree-level structures that can be glued together to produce the one-loop amplitudes. This treatment seems particularly advantageous for massless processes with high multiplicity, situations in which the corresponding number of Feynman diagrams would be very big. On the other hand, for processes with many scales and different particles, the automatized generation of amplitudes based on Feynman diagrams is still quite effective.

In both scenarios, automation is indispensable for processes with many external legs, where both the number of diagrams and the complexity of the numerators render any manual calculation unreasonable. As a first example of automation, in [49] the tree-order amplitude computation code HELAC [56], combined with the OPP reduction code CutTools, was extended to compute full one-loop amplitudes. By adding the generation of dipole terms [18], a very powerful and flexible tool for multi-leg processes emerged, that already proved its enormous potential $[4,5]$.

The process of generating the list of unintegrated amplitudes can be also automated using tools for algebraic manipulation that have been available for many years, such as QGraf [57] or Form [58]. Respect to a traditional Feynman-diagram based calculation, we should remember that the input of the integrand level reduction is the numerator of the amplitude: these functions will be only evaluated numerically at given integration momentum. We should therefore aim at compact expressions that provide the numerical value for the unintegrated numerator within a small number of operations.

Work have been already done within FeynCalc $[22,59]$ to provide 4-dimensional amplitudes that can be processed with CutTools. A different automated setup that uses QGraf, Form and haggies [60] is described in [54].

The OPP method allows for several tests on the precision of the results. In addition to standard techniques that are common to all reduction method, such as the test on the expression of the UV and IR poles or the comparison between results using different level of numerical precision, the OPP framework provides self-consistent checks on the quality of the reconstruction.

One example is the so-called " $N=N$ " test on the reconstructed numerator. The test is based on Eq.(3.1): the original numerator on the 1.h.s. and the reconstructed one on the r.h.s. should provide identical results for any value of the integration momentum. If something goes wrong with the reconstruction of the coefficients, this test will fail and the point should be discarded or reprocessed with higher-precision routines. 


\section{Conclusions and Future Outlook}

During the last year we witnessed the completion of one-loop NLO calculation of increasing complexity. Cross sections for six-particle processes with high multiplicity as well as processes with massive external and internal particles have been successfully calculated. Important results have been obtained with both improved algebraic reduction and new numerical techniques.

The OPP approach played an important role in boosting the new developments. In order to increase the level of difficulty of feasible calculations, automation becomes a fundamental requirement. Algebraic methods, after the hard work required to produce analytic compact results, provide fast calculations and good control over instabilities. In facing calculations of high complexity, it is still crucial to improve the precision and the efficiency of the new numerical algorithms.

\section{Acknowledgments}

Work supported by the NSF Grant PHY-0855489 and PSC-CUNY Award 60041-39 40. I would like to thank Roberto Pittau, Costas Papadopoulos, Andreas van Hameren, Pierpaolo Mastrolia, Michal Czakon, Stefano Actis, Andrea Ferroglia, Malgorzata Worek, Francesco Tramontano, and Thomas Reiter for fruitful collaborations, interesting discussions, and wise suggestions.

\section{References}

[1] Z. Bern et al. arXiv:0803.0494 [hep-ph]; J. R. Andersen et al. arXiv:1003.1241 [hep-ph].

[2] A. Bredenstein, A. Denner, S. Dittmaier and S. Pozzorini, Phys. Rev. Lett. 103, 012002 (2009) [arXiv:0905.0110 [hep-ph]].

[3] A. Bredenstein, A. Denner, S. Dittmaier and S. Pozzorini, arXiv:1001.4727 [hep-ph].

[4] G. Bevilacqua, M. Czakon, C. G. Papadopoulos, R. Pittau and M. Worek, JHEP 0909, 109 (2009) [arXiv:0907.4723 [hep-ph]].

[5] G. Bevilacqua, M. Czakon, C. G. Papadopoulos and M. Worek, arXiv:1002.4009 [hep-ph].

[6] C. F. Berger et al., Phys. Rev. Lett. 102, 222001 (2009) [arXiv:0902.2760 [hep-ph]].

[7] C. F. Berger et al., Phys. Rev. D 80, 074036 (2009) [arXiv:0907.1984 [hep-ph]].

[8] R. K. Ellis, K. Melnikov and G. Zanderighi, JHEP 0904, 077 (2009) [arXiv:0901.4101 [hep-ph]].

[9] R. Keith Ellis, K. Melnikov and G. Zanderighi, Phys. Rev. D 80, 094002 (2009) [arXiv:0906.1445 [hep-ph]].

[10] C. F. Berger et al., arXiv:1004.1659 [hep-ph].

[11] A. Lazopoulos, arXiv:0812.2998 [hep-ph].

[12] J. C. Winter and W. T. Giele, arXiv:0902.0094 [hep-ph].

[13] A. van Hameren, JHEP 0907, 088 (2009) [arXiv:0905.1005 [hep-ph]].

[14] R. Frederix, these proceedings.

[15] S. Catani and M. H. Seymour, Nucl. Phys. B 485, 291 (1997).

[16] S. Frixione, Z. Kunszt and A. Signer, Nucl. Phys. B 467, 399 (1996). 
[17] T. Gleisberg and F. Krauss, Eur. Phys. J. C 53, 501 (2008).

[18] M. Czakon, C. G. Papadopoulos and M. Worek, JHEP 0908, 085 (2009).

[19] R. Frederix, S. Frixione, F. Maltoni and T. Stelzer, JHEP 0910, 003 (2009).

[20] T. Diakonidis, these proceedings.

[21] T. Riemann, these proceedings.

[22] T. Hahn, arXiv:1006.2231 [hep-ph], these proceedings.

[23] D. Maitre, arXiv:1006.5594 [hep-ph], these proceedings.

[24] R. K. Ellis and G. Zanderighi, JHEP 0802, 002 (2008).

[25] G. J. van Oldenborgh, Comput. Phys. Commun. 66, 1 (1991).

[26] A. van Hameren, J. Vollinga and S. Weinzierl, Eur. Phys. J. C 41, 361 (2005).

[27] G. 't Hooft and M. J. G. Veltman, Nucl. Phys. B 153, 365 (1979).

[28] G. Passarino and M. J. G. Veltman, Nucl. Phys. B 160, 151 (1979).

[29] A. Denner and S. Dittmaier, Nucl. Phys. B 734, 62 (2006) [arXiv:hep-ph/0509141].

[30] T. Binoth, J. P. Guillet, G. Heinrich, E. Pilon and T. Reiter, Comput. Phys. Commun. 180, 2317 (2009) [arXiv:0810.0992 [hep-ph]].

[31] T. Binoth et al., arXiv:1001.4905 [hep-ph].

[32] T. Binoth, N. Greiner, A. Guffanti, J. Reuter, J. P. Guillet and T. Reiter, Phys. Lett. B 685, 293 (2010) [arXiv:0910.4379 [hep-ph]].

[33] F. Campanario, V. Hankele, C. Oleari, S. Prestel and D. Zeppenfeld, Phys. Rev. D 78, 094012 (2008) [arXiv:0809.0790 [hep-ph]].

[34] G. Bozzi, F. Campanario, V. Hankele and D. Zeppenfeld, arXiv:0911.0438 [hep-ph].

[35] Z. Bern, L. J. Dixon, D. C. Dunbar and D. A. Kosower, Nucl. Phys. B 425, 217 (1994) [arXiv:hep-ph/9403226].

[36] Z. Bern, L. J. Dixon, D. C. Dunbar and D. A. Kosower, Nucl. Phys. B 435, 59 (1995) [arXiv:hep-ph/9409265].

[37] F. del Aguila and R. Pittau, JHEP 0407, 017 (2004) [arXiv:hep-ph/0404120].

[38] G. Ossola, C. G. Papadopoulos and R. Pittau, Nucl. Phys. B 763, 147 (2007) [arXiv:hep-ph/0609007].

[39] G. Ossola, C. G. Papadopoulos and R. Pittau, JHEP 0707, 085 (2007) [arXiv:0704.1271 [hep-ph]].

[40] G. Ossola, C. G. Papadopoulos and R. Pittau, JHEP 0803, 042 (2008) [arXiv:0711.3596 [hep-ph]].

[41] C. F. Berger et al., Phys. Rev. D 78, 036003 (2008) [arXiv:0803.4180 [hep-ph]].

[42] W. T. Giele and G. Zanderighi, JHEP 0806, 038 (2008) [arXiv:0805.2152 [hep-ph]].

[43] G. Ossola, C. G. Papadopoulos and R. Pittau, Nucl. Phys. Proc. Suppl. 183, 42 (2008) and Acta Phys. Polon. B 39, 1685 (2008).

[44] G. Ossola, C. G. Papadopoulos and R. Pittau, JHEP 0805, 004 (2008) [arXiv:0802.1876 [hep-ph]].

[45] P. Draggiotis, M. V. Garzelli, C. G. Papadopoulos and R. Pittau, JHEP 0904, 072 (2009) [arXiv:0903.0356 [hep-ph]]. 
[46] M. V. Garzelli, I. Malamos and R. Pittau, JHEP 1001, 040 (2010) [arXiv:0910.3130 [hep-ph]].

[47] T. Binoth, G. Ossola, C. G. Papadopoulos and R. Pittau, JHEP 0806, 082 (2008) [arXiv:0804.0350 [hep-ph]].

[48] S. Actis, P. Mastrolia and G. Ossola, Phys. Lett. B 682, 419 (2010) [arXiv:0909.1750 [hep-ph]] and Acta Phys. Polon. B 40, 2957 (2009).

[49] A. van Hameren, C. G. Papadopoulos and R. Pittau, JHEP 0909, 106 (2009) [arXiv:0903.4665 [hep-ph]].

[50] R. K. Ellis, W. T. Giele, Z. Kunszt and K. Melnikov, Nucl. Phys. B 822, 270 (2009) [arXiv:0806.3467 [hep-ph]].

[51] R. K. Ellis, W. T. Giele and Z. Kunszt, JHEP 0803, 003 (2008) [arXiv:0708.2398 [hep-ph]].

[52] W. T. Giele, Z. Kunszt and K. Melnikov, JHEP 0804, 049 (2008) [arXiv:0801.2237 [hep-ph]].

[53] K. Melnikov and M. Schulze, arXiv:1004.3284 [hep-ph].

[54] P. Mastrolia, G. Ossola, T. Reiter and F. Tramontano, arXiv:1006.0710 [hep-ph].

[55] P. Mastrolia, G. Ossola, C. G. Papadopoulos and R. Pittau, JHEP 0806, 030 (2008) [arXiv:0803.3964 [hep-ph]].

[56] A. Kanaki and C. G. Papadopoulos, Comput. Phys. Commun. 132 (2000) 306 [arXiv:hep-ph/0002082]; A. Cafarella, C. G. Papadopoulos and M. Worek, Comput. Phys. Commun. 180, 1941 (2009) [arXiv:0710.2427 [hep-ph]].

[57] P. Nogueira, J. Comput. Phys. 105 (1993) 279-289.

[58] J. A. M. Vermaseren, arXiv:math-ph/0010025.

[59] T. Hahn, PoS ACAT08, 121 (2009) [arXiv:0901.1528 [hep-ph]].

[60] T. Reiter, Comput. Phys. Commun. 181, 1301 (2010) [arXiv:0907.3714 [hep-ph]]. 